\title{
GeSi Nanocrystals Photo-Sensors for Optical Detection of Slippery Road Conditions Combining Two Classification Algorithms
}

\author{
Catalin Palade ${ }^{1}$, Ionel Stavarache ${ }^{1, * \mathbb{C}}$, Toma Stoica ${ }^{1}$ and Magdalena Lidia Ciurea ${ }^{1,2, * \mathbb{C}}$ \\ 1 National Institute of Materials Physics, 405A Atomistilor Street, 077125 Magurele, Romania; \\ catalin.palade@infim.ro (C.P.); toma.stoica@infim.ro (T.S.) \\ 2 Academy of Romanian Scientists, 54 Splaiul Independentei, 050094 Bucharest, Romania \\ * Correspondence: stavarache@infim.ro (I.S.); ciurea@infim.ro (M.L.C.)
}

Received: 30 September 2020; Accepted: 5 November 2020; Published: 9 November 2020

check for updates

\begin{abstract}
One of the key elements in assessing traffic safety on the roads is the detection of asphalt conditions. In this paper, we propose an optical sensor based on GeSi nanocrystals embedded in $\mathrm{SiO}_{2}$ matrix that discriminates between different slippery road conditions (wet and icy asphalt and asphalt covered with dirty ice) in respect to dry asphalt. The sensor is fabricated by magnetron sputtering deposition followed by rapid thermal annealing. The photodetector has spectral sensitivity in the $360-1350 \mathrm{~nm}$ range and the signal-noise ratio is $10^{2}-10^{3}$. The working principle of sensor setup for detection of road conditions is based on the photoresponse (photocurrent) of the sensor under illumination with the light reflected from the asphalt having different reflection coefficients for dry, wet, icy and dirty ice coatings. For this, the asphalt is illuminated sequentially with 980 and $1064 \mathrm{~nm}$ laser diodes. A database of these photocurrents is obtained for the different road conditions. We show that the use of both k-nearest neighbor and artificial neural networks classification algorithms enables a more accurate recognition of the class corresponding to a specific road state than in the case of using only one algorithm. This is achieved by comparing the new output sensor data with previously classified data for each algorithm and then by performing an intersection of the algorithms' results.
\end{abstract}

Keywords: optical sensor; photodetection of reflected light from asphalt; road conditions detection sensor; road safety; smart roads; k-nearest neighbor algorithm; artificial neural networks

\section{Introduction}

The car manufacturers, together with road authorities, are continuously challenged to supply effective services to ensure efficient and safe transportation. Fast and correct evaluation of slippery road conditions is critical for a driver, who should evaluate the conditions in order to have safe transportation and to prevent traffic accidents. The slow response of a human being is due to the limited information about road conditions that the human eye can provide and, therefore, using sensors for discriminating between dry, wet or icy asphalt is a requirement for the mitigation of traffic accidents.

At present, different types of technological systems, like fixed automated spray technology (FAST) [1,2], road weather information systems (RWIS) [3,4], environmental sensors [5], sensor networks for smart roads [6,7], pavement surface temperature sensors [8] and modern weather forecast systems [9], have been developed for detecting the asphalt state or for road monitoring under different atmospheric conditions. Great efforts have been made to integrate all these technological systems into a cooperative-intelligent transportation system (C-ITS) [10]. This platform was proposed to facilitate communications between vehicles and vehicles (V2V), vehicles and infrastructure (V2I) and infrastructure and vehicles (I2V) [11,12]. 
The development of sensor-based systems is the most appropriate solution with flexibility, portability and high applicability, which overcomes the limitations of the fixed systems as RWIS. Different sensor approaches were proposed in the study of Tabatabai et al. in which the authors proposed a sensor based on electrical resistance measurements that, besides determining roadway conditions (icy, dry, wet and frozen), transmits warnings about location and surface temperature [13]. Piccardi et al. studied different sensing geometries for the evaluation of the asphalt state using the measurement of the polarization/depolarization state of near infrared radiation (by two photodiodes). From their results, one can distinguish between a safe surface and different dangerous surfaces (wet, with water and icy) [14]. Jonsson et al. proposed another technique for determining road conditions based on infrared thermometry [15]. The measurements performed with a capacitive sensor represent another alternative proposed for detection of the road surface covered with water or ice. The limitation of this method is related to sensor contamination with dirt, fuel and salt [16-18]. In the paper published by Alimasi et al., the efforts are focused on a portable measurement system that can distinguish between different road states by comparing the ratio between specular reflectance and diffuse reflectance [19]. The recognition based on video images for identifying road conditions represents another approach proposed by Zhao et al. This method is based on road surface state recognition using a support vector machine (SVM) sustained by a grid searching algorithm and a particle swarm optimization algorithm (PSO) to improve recognition accuracy [20].

Using optical sensors to discriminate between different slippery road conditions is one of the best solution. Such an optical system is usually based on the comparison of the reflection coefficient at different wavelengths for different road states [14,15,21-24], for which broad-band optical sensors with extended sensitivity from visible (VIS) to short wave infrared (SWIR, 1-3 $\mu \mathrm{m}$ ) are needed. Group IV, Si-Ge-Sn based photodetectors are very promising non-toxic alternatives to market available III-V devices. A cost-effective technique based on SiGeSn nanocrystals (NCs) embedded in an oxide matrix obtained by magnetron sputtering can be used for large-scale production of highly sensitive VIS-SWIR optical sensors [25-30]. In such composite materials, the oxide matrix has the role of the surface passivation of nanocrystals $[28,29,31-33]$. SiGe NCs alloys have the advantage of tuning the energy bandgap by adjusting the SiGe composition, in addition to the size controlling of the bandgap by quantum confinement effect. The $\mathrm{SiGeSiO}_{2}$ amorphous films deposited by magnetron sputtering and successively annealed by rapid thermal annealing (RTA) within the $700-1000{ }^{\circ} \mathrm{C}$ range for NC self-assembly, have shown photoresponsivity of about $5 \mathrm{AW}^{-1}$ at room temperature, measured on coplanar diode structures [28].

In this paper we describe an optimized new optical sensor based on GeSi NCs embedded in a $\mathrm{SiO}_{2}$ matrix, that are fabricated by magnetron co-sputtering deposition of $\mathrm{Ge}, \mathrm{Si}$ and $\mathrm{SiO}_{2}$ followed by thermal annealing, to be used for discriminating between different slippery road conditions in respect to dry asphalt. Such sensors based on eco-friendly materials, compatible with the well-established technology of $\mathrm{Si}$, using cost-effective fabrication technology have many socio and economic advantages in respect to other sensors available on the market. The GeSi NCs sensors were experimentally tested by using them within an optical set-up for the detection of four types of road surface, namely dry, wet, icy asphalt and dirty ice asphalt. The experimental data were processed by two classification algorithms, namely k-nearest neighbor (KNN) and artificial neural network (ANN) algorithms for identifying the class to which road condition belonged. An important advantage of the proposed sensor is that this is also completely customizable for a better fit with the required needs. Thus, by changing the fabrication parameters, the spectral sensitivity of the sensor was adjusted in order to achieve a better matching between spectral characteristics of dry, wet and icy asphalt and the sensitivity domain of the sensor. 


\section{Materials and Methods}

\subsection{Optical Sensor Fabrication}

The films with $\mathrm{GeSiSiO}_{2}$ were deposited by magnetron sputtering. The fabrication process was described elsewhere $[27,28]$. Here, we describe the layer fabrication procedure for optimized annealing. Thus, three separate targets $\left(\mathrm{Ge}, \mathrm{Si}\right.$ and $\left.\mathrm{SiO}_{2}\right)$ under DC and RF regime (20 W DC for Ge, $38 \mathrm{~W}$ DC for Si

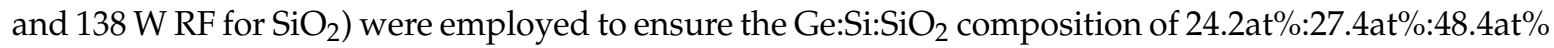
(corresponding to $\mathrm{Si}_{x} \mathrm{Ge}_{1-x}$ alloy in $\mathrm{SiO}_{2}$, with a concentration of $\mathrm{x}=53 \mathrm{at} \%$ ). The deposition was made on n-type Si wafers chemically cleaned by a standard RCA(Radio Corporation of America) method in a Piranha solution and then covered by $50 \mathrm{~nm} \mathrm{SiO}$ grown by dry oxidation in a rapid thermal processor (RTP) for electrical isolation of the GeSi NCs active layer from Si substrate. The as-deposited films have about $320 \mathrm{~nm}$ thickness.

The GeSi nanocrystallization was made by rapid thermal annealing (RTA) at optimal temperature of $900{ }^{\circ} \mathrm{C}$ for $10 \mathrm{~min}$ in $\mathrm{N}_{2}$ atmosphere. RTA temperature and time are critical in obtaining photosensitive GeSi NCs due to the competition between Ge fast diffusion and GeSi NCs formation in $\mathrm{SiO}_{2}$ matrix [25-28].

The workflow for obtaining the GeSi NCs:SiO $2 / \mathrm{SiO}_{2} / \mathrm{n}-\mathrm{Si}$ photodetector with $\mathrm{Al}$ contacts (magnetron sputtering) in planar geometry is presented in Figure 1.

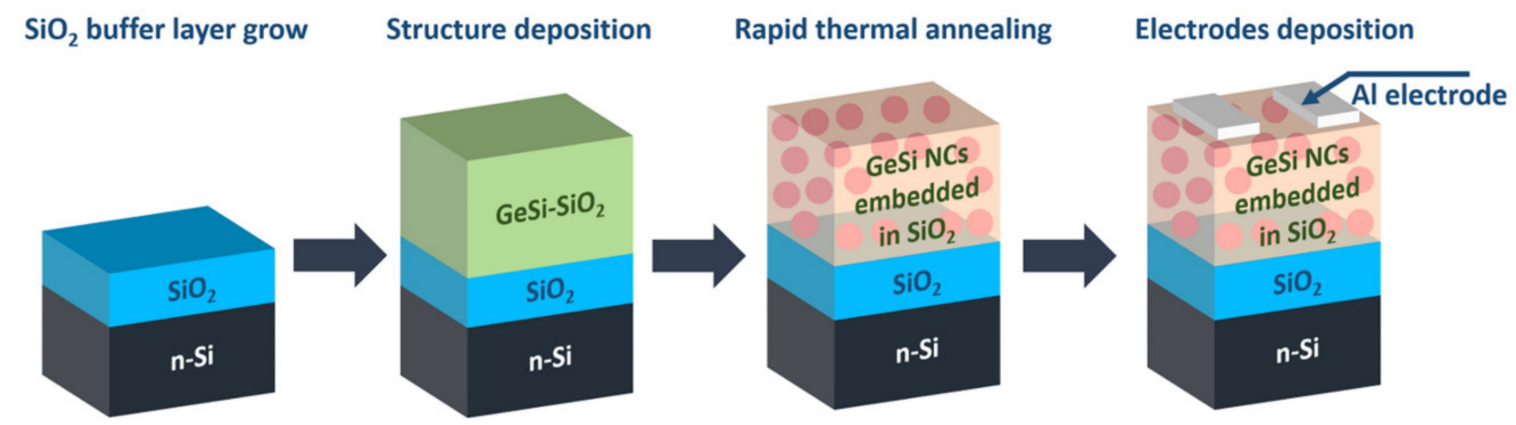

Figure 1. The workflow for obtaining the GeSi NCs:SiO $2 / \mathrm{SiO}_{2} / \mathrm{n}-\mathrm{Si}$ photodetector.

\subsection{Sensor Signal Measurements and Software Packet for Data Analysis}

The spectral dependence of the sensor sensitivity was measured under monochromatic illumination with modulated light (120 Hz frequency) using an incandescent lamp (250 W) and Newport monochromator. For this, a lock-in amplifier (SR830) and a mechanical chopper (SR540) were used. For acquiring the photo-signal data for different states of the asphalt, the monochromatic light emitted by pulsed laser diodes of different wavelengths was measured after diffuse reflection on the asphalt surface using a homemade data acquisition system described below. For database analysis by discrimination algorithms, the Python 3.7 programming language was used. The software source codes and resulting data corresponding to the used KNN, ANN and comparing algorithms are given by Supplementary Materials.

\section{Results and Discussions}

\subsection{Photodetector Characterization}

Figure 2 shows the cross-section TEM image at low magnification of GeSi NCs: $\mathrm{SiO}_{2} / \mathrm{SiO}_{2} / \mathrm{n}-\mathrm{Si}$ structure. The image reveals the increase in density and the decrease in the size of the NCs towards the $\mathrm{SiO}_{2} / \mathrm{Si}$ substrate. This non-uniformity is caused by Ge and $\mathrm{Si}$ segregation and diffusion processes influenced by the depth-dependent internal stress in the film [34]. The upper part of the film of about $85 \mathrm{~nm}$ shows low Ge concentration due to the fast Ge diffusion and its surface oxidation and evaporation. By construction, the $\mathrm{GeSi} \mathrm{NCs}: \mathrm{SiO}_{2}$ layer is electrically isolated from the Si substrate by a $50 \mathrm{~nm}$ of $\mathrm{SiO}_{2}$ buffer layer. 


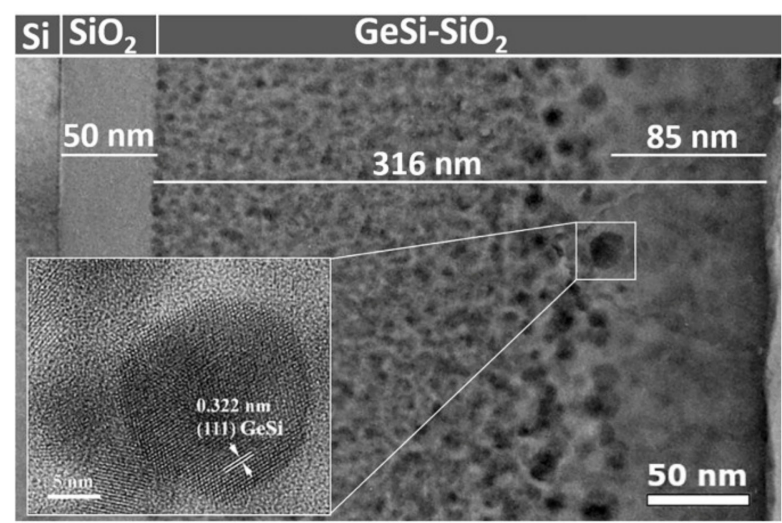

Figure 2. Low magnification XTEM image of GeSi NCs:SiO $2 / \mathrm{SiO}_{2} / \mathrm{n}-\mathrm{Si}$ structure. Inset is an HRTEM image of a spherical GeSi NC.

The size of GeSi NCs increases from $5 \mathrm{~nm}$ at the bottom of the film to $18 \mathrm{~nm}$ at the surface. Figure 2 shows a low magnification XTEM (Cross-Sectional Transmission Electron Microscopy) image of the GeSi NCs: $\mathrm{SiO}_{2}$ film on $\mathrm{SiO}_{2} / \mathrm{n}$-Si substrate. The inset in Figure 2 shows the HRTEM (High-Resolution Transmission Electron Microscopy) image of a spherical GeSi NC. The distance of $0.322 \mathrm{~nm}$ between (111) planes in GeSi NCs corresponds to about 40at $\% \mathrm{Si}$ concentration as evaluated by linear interpolation, neglecting possible influence of strain in NCs. This value of Si concentration in GeSi NCs is lower than the mean concentration of about 53 at $\% \mathrm{Si}$ of the SiGe alloy in the fabricated film.

The non-uniform distribution of the NCs size is beneficial for broadening of the photosensitivity spectra. Figure 3 shows the photocurrent spectrum of the photodetector with spectral sensitivity detected in the range of $360-1350 \mathrm{~nm}$.

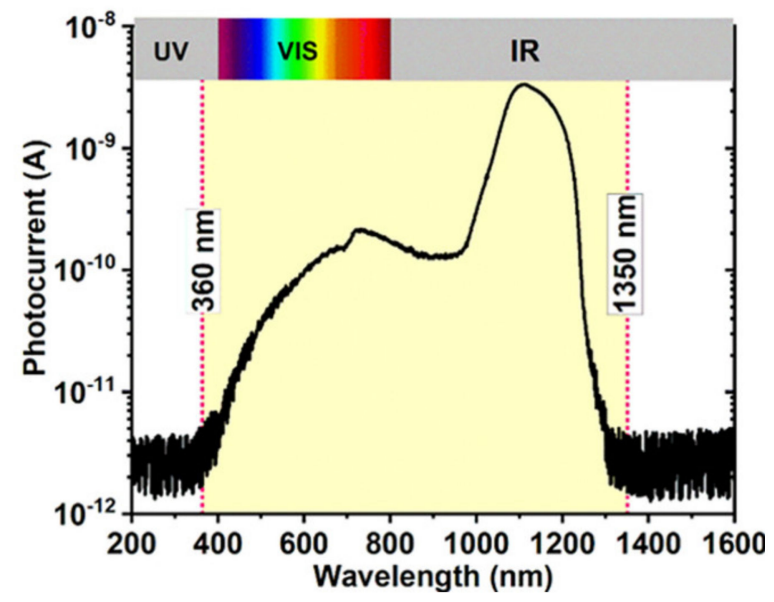

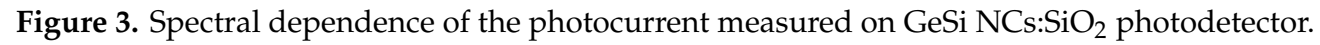

The spectral distribution curve of the photocurrent presents a maximum at $1100 \mathrm{~nm}$ wavelength and another broad one in the $600-1000 \mathrm{~nm}$ range. The signal-noise ratio is $10^{2}-10^{3}$.

\subsection{Data Acquisition System}

The working principle of the proposed optical sensor setup is based on different specular/diffuse reflections of the dry, wet and icy asphalt at different illumination wavelengths that is sketched in Figure $4 \mathrm{a}$. Figure $4 \mathrm{~b}$ shows the workflow of the sensor setup. 
(a)

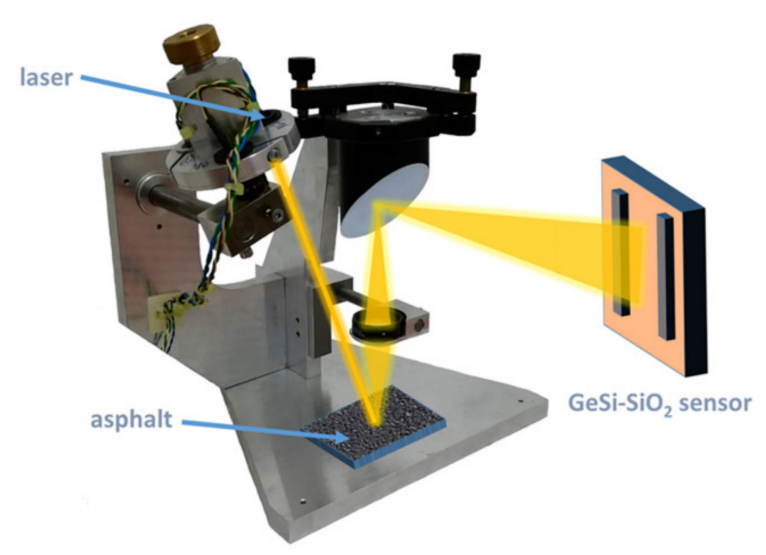

(b)

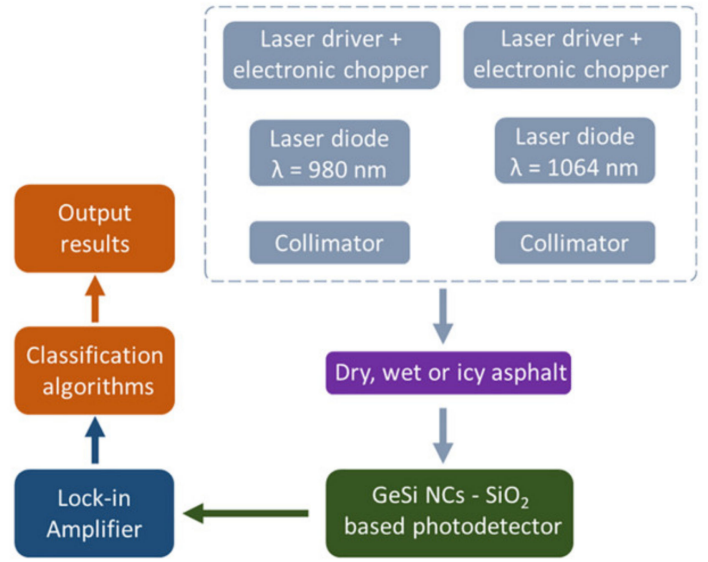

Figure 4. (a) The working principle and (b) the workflow of the sensor setup.

Based on the spectral dependence of the photoresponse of $\mathrm{GeSi} N \mathrm{NCs}: \mathrm{SiO}_{2}$ photodetector, we chose as the illumination source two laser diodes emitting about $200 \mathrm{~mW}$ in near-infrared (NIR), one laser diode RLT980-150GS of $150 \mathrm{~mW}$ from Roithner Lasertechnik with a wavelength of $\lambda=980 \mathrm{~nm}$ and another laser diode M9-A64-0200 of $200 \mathrm{~mW}$ from Thorlabs with a wavelength of $\lambda=1064 \mathrm{~nm}$. The system is designed to be placed along the roads using an electric grid or independent renewable energy sources but could also be easily implemented in a novel integrated topology for electric vehicles [35]. The laser light and specular/diffuse reflections of light from the asphalt is collimated by using a collimator with a quartz lens and a parabolic mirror as shown in the Figure 4a. The power supply for the laser diodes was provided from a custom made constant current source circuit using a LM317 integrated circuit. The current was modulated through a BD139 transistor by using a TTL (Transistor-Transistor Logic) signal $(0 \div 5 \mathrm{~V})$ from an Arduino UNO board with a modulation frequency of $119 \mathrm{~Hz}$. The electric circuit of the laser diode power supply is presented in Figure 5.

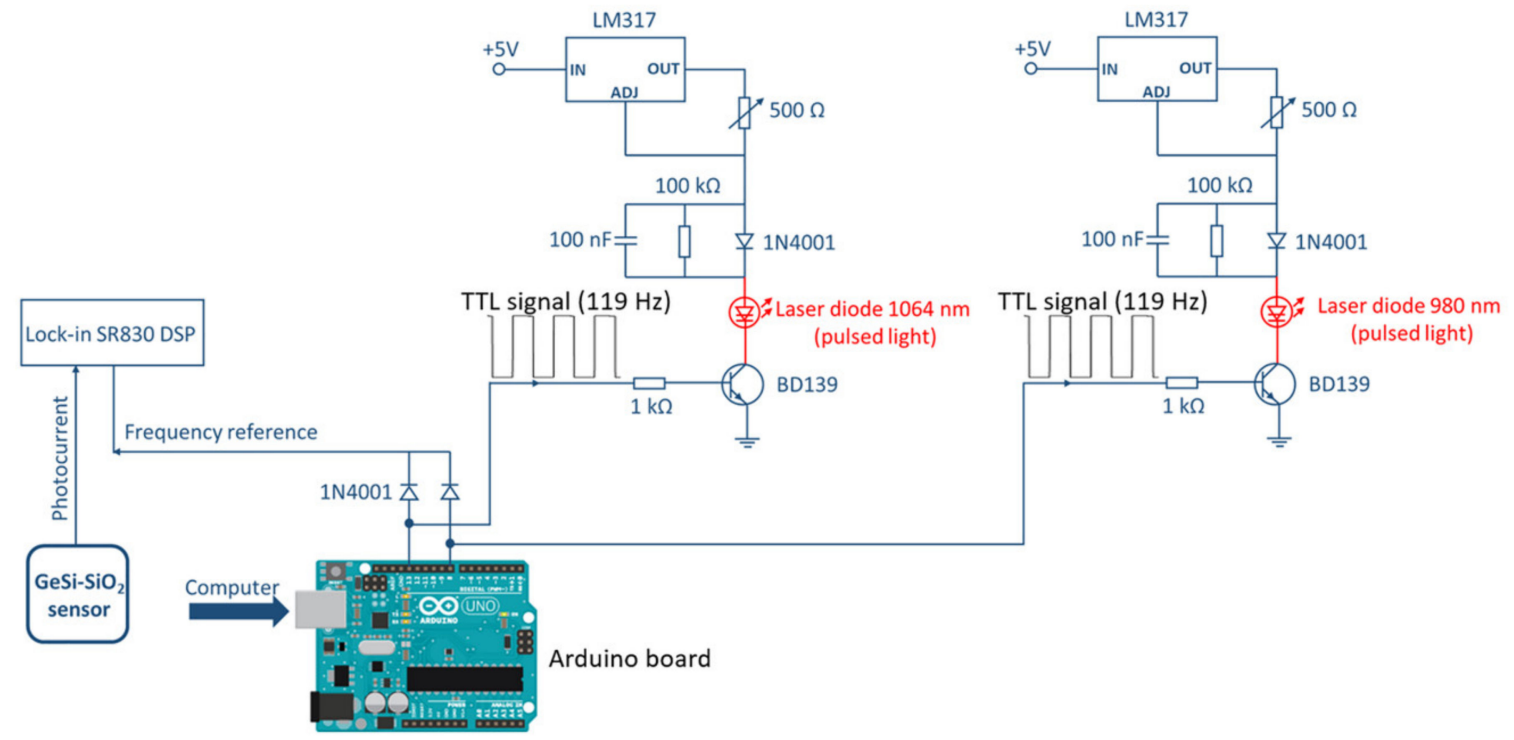

Figure 5. The electric circuit of the laser diode power supply.

By modulating the current in the laser power supply, a pulsed laser light was obtained with the frequency of the TTL signal. The TTL signals measured by an oscilloscope are generated by an Arduino board and illustrated in Figure 5. Each laser diode has a separate power supply connected to the same Arduino board which gives the possibility to separately control the laser diodes from a computer 
connected to the Arduino board. The measurement procedure consists of the successive illumination of the asphalt at a precise angle with the two laser diodes such that only one laser diode is active at a time, then the reflected light is detected by the GeSi NCs:SiO 2 photodetector. The photocurrent generated by the reflected light is then measured with a Stanford SR830 DSP lock-in amplifier that uses the frequency reference of the TTL signal generated by the Arduino board.

\subsection{Optoelectronic Database for Different Asphalt States}

One complete measurement is done when the asphalt is successively illuminated with the two laser diodes. The photocurrent $I_{1064}$, generated by the $\lambda=1064 \mathrm{~nm}$ wavelength illumination, is plotted as a function of the photocurrent $I_{980}$, generated by the $\lambda=980 \mathrm{~nm}$ illumination. So, a complete determination represents one point in the $I_{1064}-I_{980}$ plot. The experimental results in Figure 6 were obtained by multiple measurements (in different zones of the asphalt) of the photocurrent for the two laser illumination for each case of dry, wet, icy asphalt and dirty ice (a mixture of asphalt powder, dust and water, frozen together). The asphalt was illuminated at an angle of 30 degrees related to normal incidence (Figure 4a). One can see that there is a good separation between the results corresponding to the different states of asphalt.

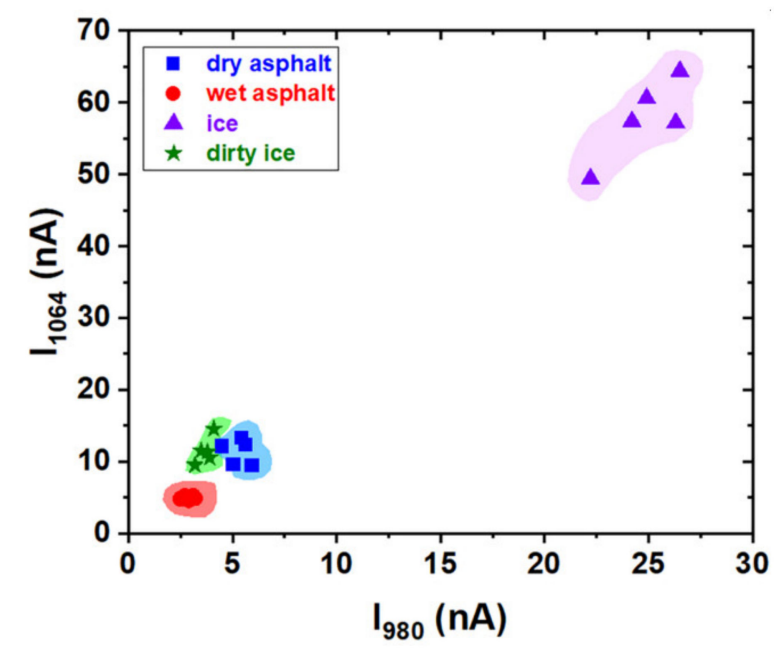

Figure 6. Experimental results obtained by multiple measurements of the photocurrent for the two laser diode illumination in the case of dry, wet, icy asphalt and dirty ice (frozen monolith of mixed asphalt powder, dust and water).

The best separation is obtained for icy asphalt for which one can observe that the photocurrent for both wavelengths $(\lambda=980 \mathrm{~nm}$ and $\lambda=1064 \mathrm{~nm})$ is with order of magnitude higher than the photocurrent that corresponds to wet asphalt. In the case of dry asphalt, illumination with the $\lambda=980$ nm wavelength generates a higher photocurrent compared with the one for dirty ice.

\subsection{Database Analysis by Discrimination Algorithms}

In order to have a system for complete detection of road conditions, it should be able to discriminate between dry, wet or icy asphalt. For this, first of all, separable clusters/classes of output data from the sensor $\left(\left(I_{1064}, I_{980}\right)\right.$ points), corresponding to each type of asphalt (dry, wet, icy asphalt and dirty ice), should be obtained. Additionally, it is necessary to have a classification algorithm to recognize the class by comparing the new output sensor data with previously classified data. For this, we used two different types of classification algorithms, namely k-nearest neighbor algorithm (KNN) and artificial neural network algorithm (ANN) [36]. For programming these algorithms, the Python 3.7 programming language was used and for the ANN algorithm, additionally, the Scikit-learn python [37,38] library was used. The KNN algorithm calculates the Euclidian distances in the $I_{1064}-I_{980}$ plane between the 
point that corresponds to new output sensor data and each point already classified from the measured photocurrents database and, finally, chooses the class to which the new point belongs considering the minimum distance (Figure 7a). We applied the KNN algorithm to our experimental data by generating an array of $30 \times 70$ points in the $0-30 \mathrm{nA}$ and $0-70 \mathrm{nA}$ ranges and classified them in Figure $7 \mathrm{~b}$.

(a)
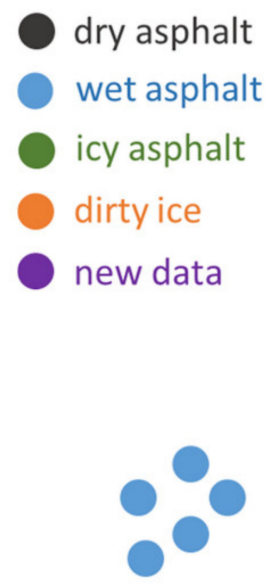

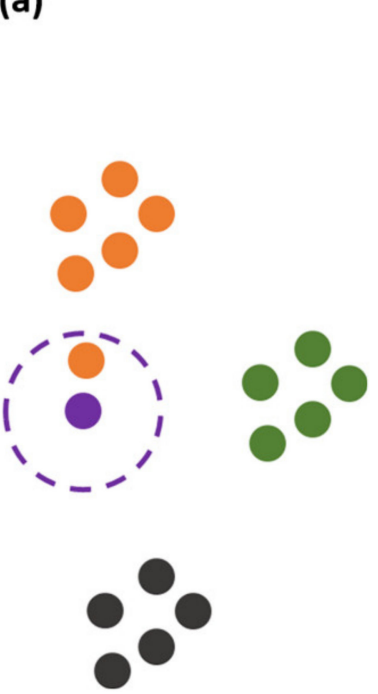

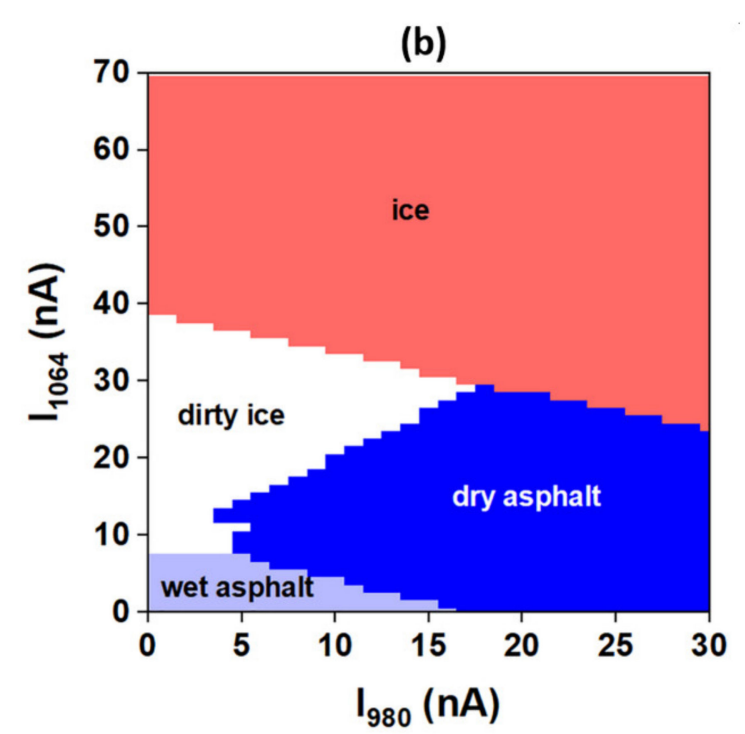

Figure 7. K-nearest neighbor (KNN) algorithm: (a) the working principle and (b) KNN algorithm applied to array data.

The advantage of the KNN algorithm over the ANN is that the KNN algorithm requires no training before using it in the classification procedure. However, the KNN drawback is that at higher database size the classification process becomes slower due to more iterations involved. Alternative fuzzy logic that is not investigated here could be based on the field programmable gate array (FPGA) or its Proportional-Integral-Derivative (PID)-type version [39]. The ANN algorithm works differently by simulating a network of artificial neurons that requires a training procedure [37] that is time consuming but at the end of the training procedure the algorithm works without training data making the classification very fast. We also applied the ANN algorithm to our experimental data, similarly to KNN algorithm. The proposed ANN configuration consists of two input neurons, four hidden layers (each one with 28 neurons) and four output neurons, the total number being 2520 synapses (weights). Each neuron in the network uses a rectifier linear unit (ReLU) as an activation function. The ANN algorithm was trained using the experimental data plotted in Figure 6 and after training, the network was fed with the same array as for the KNN algorithm. The results of applying the ANN algorithm on the array data and the working principle of the algorithm are presented in Figure 8.

Both KNN and ANN algorithms provide a good mapping of the array data (data that arrive from the sensor). However, both algorithms have as a drawback the permanent correspondence between new data and the asphalt state. In other words, new data will always be assigned to one of the dry, wet, icy asphalt and dirty ice classes which is not always a correct decision due to the possibility of an asphalt mixed state occurring like wet-ice-dry asphalt or a new asphalt condition that needs to be learned. This drawback can be overcome by limiting the algorithms' mapping around the experimental data and by introducing a new class that corresponds to undecided action which triggers the learning procedure of the ANN algorithm in order to assign the undecided point to an existing class or to create another one. This can be done by intersection of the classification results of the KNN and ANN algorithms. This means that the new data can be assigned to an existing class only if it is confirmed by both algorithms. If the asphalt class is confirmed by only one algorithm, then it will be assigned to an undecided class. The results of the algorithms' intersection are presented in Figure 9. The software 
source codes and the resulting data corresponding to the KNN, ANN and comparing algorithms are given in the Supplementary Materials.

(a)

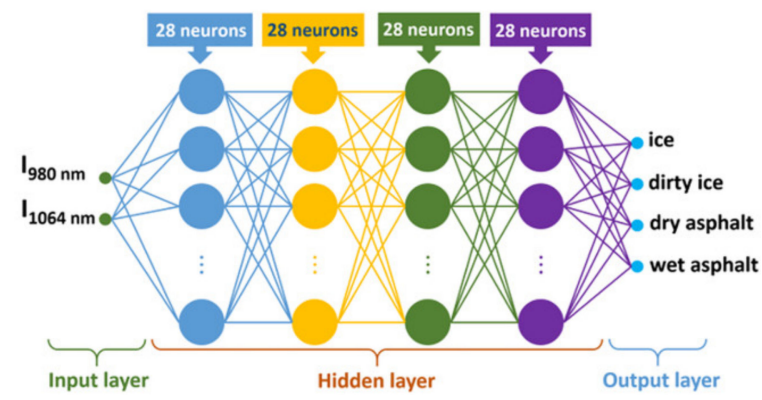

(b)

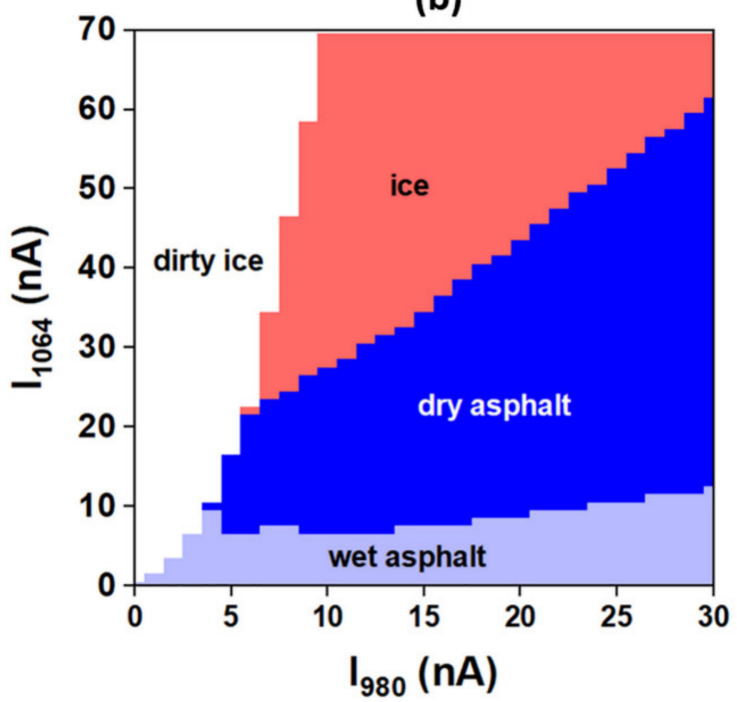

Figure 8. Artificial neural network (ANN) algorithm: (a) the working principle and (b) ANN algorithm applied to array data.

(a)

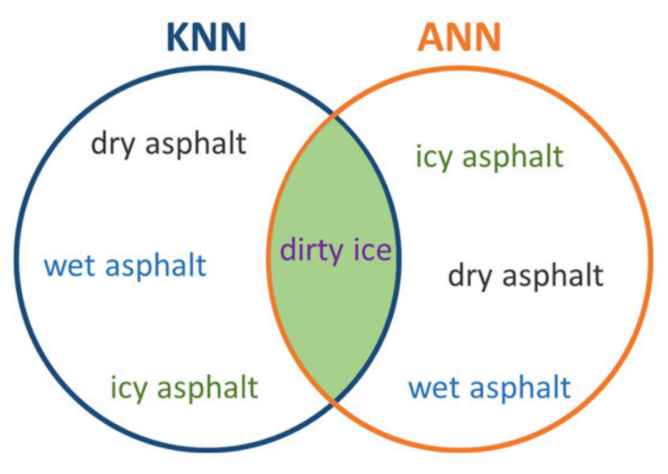

(b)

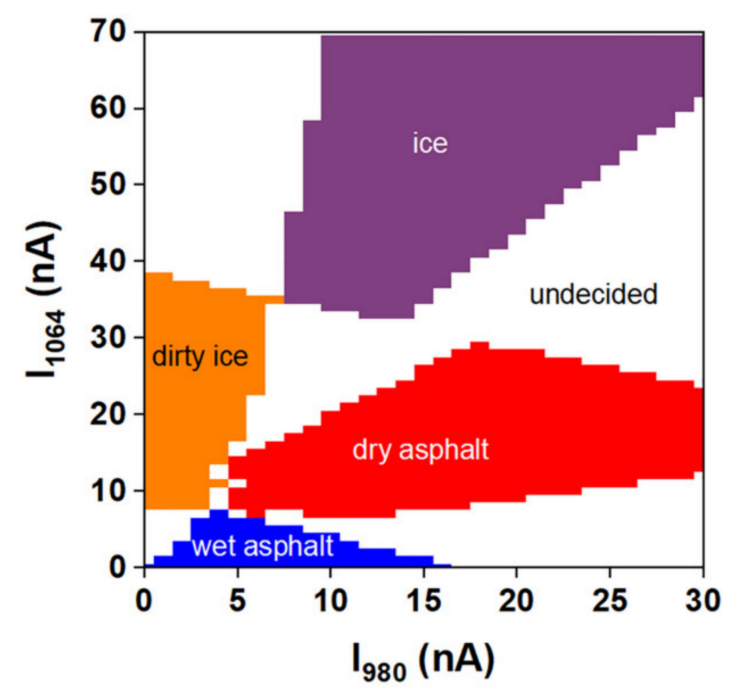

Figure 9. KNN and ANN algorithms' classification intersection: (a) a schematic example of the intersection of the classification results of the KNN and ANN algorithms for the dirty ice state and (b) results of KNN and ANN intersection applied on the array data.

In reality, the undecided results correspond to an asphalt mixed state or to an unknown state which need to be covered by classification algorithms by labeling the data and training the algorithms. If a classification algorithm is trained to recognize a certain state of the asphalt, then the undecided results can be ignored because it is less probable for it to correspond to that specific state for which the algorithm is trained to recognize. 


\section{Conclusions}

An optical sensor system based on $\mathrm{GeSi}$ NCs embedded in an $\mathrm{SiO}_{2}$ matrix was developed for discriminating between different slippery road conditions, namely wet, icy asphalt and dirty ice (frozen monolith of mixed asphalt powder, dust and water) in respect to dry asphalt. The sensor was fabricated by magnetron sputtering deposition of $\mathrm{Ge}, \mathrm{Si}$ and $\mathrm{SiO}_{2}$ on oxidized n-type Si substrate, followed by RTA annealing for GeSi NCs formation. The photodetector has a spectral sensitivity in the range of $360-1350 \mathrm{~nm}$ and the signal-noise ratio is $10^{2}-10^{3}$. The working principle of the sensor setup is based on the different reflection coefficients of the dry, wet and icy asphalt illuminated with 980 and $1064 \mathrm{~nm}$ laser diodes, one at a time. The experimental results show that the data obtained for different asphalt states present good separation and that it is possible to use classification algorithms, such as the k-nearest neighbor and artificial neural networks employed by us. Each classification algorithm provides excellent overlapping between experimental data and classified (predicted) ones but the mapping prediction falsely extends to infinity. This limitation is overcome by using the intersection of the classification results of the KNN and ANN algorithms leading to the constraining of the mapping prediction near the experimental data. The optical sensor together with the setup to be mounted along the road for road safety conditions is dedicated to be a potential platform for warning drivers with enough time and distance before reaching the slippery road. The proposed setup involves cheap materials, electronic components and fabrication processes. For future development of real cost-effective applications of the proposed road state detection platform, cheap light emitting diodes can be used. The present experiments have used lasers of less than $200 \mathrm{~mW}$ power, emitting in near infrared. Currently, the system is designed to be placed along the roads, ideally using renewable energy sources, but the proposed electro-optic detection system could be easily implemented in the future in a novel integrated topology for electric vehicles.

Supplementary Materials: Software source codes and resulting data corresponding to the used KNN, ANN and comparing algorithms are available online at http://www.mdpi.com/1424-8220/20/21/6395/s1.

Author Contributions: Conceptualization, all authors; software, C.P.; investigation, C.P., I.S. and T.S.; writing-original draft preparation, C.P. and I.S.; writing-review and editing, T.S. and M.L.C.; supervision, M.L.C. All authors have read and agreed to the published version of the manuscript.

Funding: This research was funded by UEFISCDI, project M-ERA.NET PhotoNanoP Contract No. 33/2016 and by the Romanian Ministry of Research and Innovation, NIMP Core Program PN19-03 Contract no. 21N/2019.

Acknowledgments: The authors gratefully acknowledge partners from Optoelectronica 2001 company: Gheorghe Stan for his contribution to the design of the optoelectronic setup and its testing and Alexandra Enuica for her contribution to the supervision of the project activity.

Conflicts of Interest: The authors declare no conflicts of interest.

\section{References}

1. Ye, Z.; Wu, J.; Ferradi, N.E.; Shi, X. Anti-icing for key highway locations: Fixed automated spray technology. Can. J. Civ. Eng. 2013, 40, 11-18. [CrossRef]

2. Yang, C.H.; Kim, J.G.; Yun, D.G.; Sung, J.G. Prioritization of the fixed automated spray system implementation (South Korean experience). KSCE J. Civ. Eng. 2017, 22, 1946-1953. [CrossRef]

3. Zhao, L.; Chien, S.I.; Liu, X.; Liu, W. Planning a road weather information system with GIS. J. Mod. Transp. 2015, 23, 176-188. [CrossRef]

4. Kwon, T.J.; Fu, L.; Melles, S.J. Location optimization of road weather information system (RWIS) network considering the needs of winter road maintenance and the traveling public. Comput. Aided Civ. Infrastruct. Eng. 2016, 32, 57-71. [CrossRef]

5. Shibata, Y.; Arai, Y.; Saito, Y.; Hakura, J. Development and evaluation of road state information platform based on various environmental sensors in snow countries. In Advances in Internet, Data and Web Technologies EIDWT 2020 Lecture Notes on Data Engineering and Communications Technologies; Barolli, L., Okada, Y., Amato, F., Eds.; Springer: Cham, Switzerland, 2020; Volume 47. 
6. Gallo, M.; De Luca, G. Spatial extension of road traffic sensor data with artificial neural networks. Sensors 2018, 18, 2640. [CrossRef] [PubMed]

7. Masini, B.; Bazzi, A.; Zanella, A. A Survey on the roadmap to mandate on board connectivity and enable V2V-based vehicular sensor networks. Sensors 2018, 18, 2207. [CrossRef]

8. Ye, Z.; Wang, L.; Xu, W.; Gao, Z.; Yan, G. Monitoring traffic information with a developed acceleration sensing node. Sensors 2017, 17, 2817. [CrossRef] [PubMed]

9. Siems-Anderson, A.R.; Walker, C.L.; Wiener, G.; Mahoney, W.P.; Haupt, S.E. An adaptive big data weather system for surface transportation. Transp. Res. Interdiscip. Perspect. 2019, 3, 100071. [CrossRef]

10. Padarthy, M.; Heyns, E. Identification and classification of slippery winter road conditions using ommonly available vehicle variables. Transp. Res. Rec. J. Transp. Res. Board 2019, 2673, 60-70. [CrossRef]

11. Dey, K.C.; Rayamajhi, A.; Chowdhury, M.; Bhavsar, P.; Martin, J. Vehicle-to-vehicle (V2V) and vehicle-to-infrastructure (V2I) communication in a heterogeneous wireless network-Performance evaluation. Transp. Res. Part C Emerg. Technol. 2016, 68, 168-184. [CrossRef]

12. Pochettino, O.; Kondapalli, S.H.; Aono, K.; Chakrabartty, S. Real-time infrastructure-to-vehicle communication using RF-triggered wireless sensors. In Proceedings of the IEEE 62nd International Midwest Symposium on Circuits and Systems (MWSCAS), Dallas, TX, USA, 4-7 August 2019; pp. 556-559.

13. Tabatabai, H.; Aljuboori, M. A Novel concrete-based sensor for detection of ice and water on roads and bridges. Sensors 2017, 17, 2912. [CrossRef]

14. Piccardi, A.; Colace, L. Optical detection of dangerous road conditions. Sensors 2019, 19, 1360. [CrossRef]

15. Jonsson, P.; Riehm, M. Infrared thermometry in winter road maintenance. J. Atmos. Ocean. Technol. 2012, 29, 846-856. [CrossRef]

16. Troiano, A.; Pasero, E.; Mesin, L. New system for detecting road ice formation. IEEE Trans. Instrum. Meas. 2011, 60, 1091-1101. [CrossRef]

17. Baby, K.C.; George, B. A Capacitive ice layer detection system suitable for autonomous inspection of runways using an ROV. In Proceedings of the IEEE International Symposium on Robotic and Sensors Environments Proceedings, Magdeburg, Germany, 16-18 November 2012; pp. 127-132.

18. Döring, J.; Tharmakularajah, L.; Happel, J.; Krieger, K.-L. A Novel approach for road surface wetness detection with planar capacitive sensors. J. Sens. Sens. Syst. 2019, 8, 57-66. [CrossRef]

19. Alimasi, N.; Takahashi, S.; Enomoto, H. Development of a mobile optical system to detect road-freezing conditions. Bull. Glaciol. Res. 2012, 30, 41-51. [CrossRef]

20. Zhao, J.; Wu, H.; Chen, L. Road surface state recognition based on SVM optimization and image segmentation processing. J. Adv. Transp. 2017, 2017, 6458495. [CrossRef]

21. Aguilar, J.J.C.; Carrillo, J.A.C.; Fernández, A.J.G.; Acosta, E.C. Robust road condition detection system using in-vehicle standard sensors. Sensors 2015, 15, 32056-32078. [CrossRef]

22. Casselgren, J.; Sjödahl, M.; LeBlanc, J. Angular spectral response from covered asphalt. Appl. Opt. 2007, 46, 4277-4288. [CrossRef]

23. Ogura, T.; Kageyama, I.; Nasukawa, K.; Miyashita, Y.; Kitagawa, H.; Imada, Y. Study on a road surfacesensing system for snow and ice road. JSAE Rev. 2002, 23, 333-339. [CrossRef]

24. Meng, L.; Wang, L.; Hou, Y.; Yan, G. A Research on low modulus distributed fiber optical sensor for pavement material strain monitoring. Sensors 2017, 17, 2386. [CrossRef]

25. Teodorescu, V.S.; Ghica, C.; Maraloiu, A.V.; Vlaicu, M.; Kuncser, A.; Ciurea, M.L.; Stavarache, I.; Lepadatu, A.M.; Scarisoreanu, N.D.; Andrei, A.; et al. Nanostructuring of GeTiO amorphous film by pulsed laser irradiation. Beilstein J. Nanotechnol. 2015, 6, 893-900. [CrossRef]

26. Slav, A.; Palade, C.; Lepadatu, A.M.; Ciurea, M.L.; Teodorescu, V.S.; Lazanu, S.; Maraloiu, A.V.; Logofatu, C.; Braic, M.; Kiss, A. How morphology determines the charge storage properties of Ge nanocrystals in $\mathrm{HfO}_{2}$. Scripta Mater. 2016, 113, 135-138. [CrossRef]

27. Stavarache, I.; Logofatu, C.; Sultan, M.T.; Manolescu, A.; Svavarsson, H.G.; Teodorescu, V.S.; Ciurea, M.L. SiGe Nanocrystals in $\mathrm{SiO}_{2}$ with high photosensitivity from visible to short-wave infrared. Sci. Rep. 2020, 10, 3252. [CrossRef]

28. Ciurea, M.L.; Stavarache, I.; Lepadatu, A.-M.; Pasuk, I.; Teodorescu, V.S. Electrical properties related to the structure of GeSi nanostructured films. Physica Status Solidi B 2014, 251, 1340-1346. [CrossRef] 
29. Slav, A.; Palade, C.; Logofatu, C.; Dascalescu, I.; Lepadatu, A.-M.; Stavarache, I.; Comanescu, F.; Iftimie, S.; Antohe, S.; Lazanu, S.; et al. GeSn nanocrystals in $\mathrm{GeSnSiO}_{2}$ by magnetron sputtering for short- wave infrared detection. ACS Appl. Nano Mater 2019, 2, 3626-3635. [CrossRef]

30. Dascalescu, I.; Zoita, N.; Slav, A.; Matei, E.; Iftimie, S.; Comanescu, F.; Lepadatu, A.-M.; Palade, C.; Lazanu, S.; Buca, D.; et al. Epitaxial GeSn obtained by high power impulse magnetron sputtering and the heterojunction with embedded GeSn nanocrystals for SWIR detection. ACS Appl. Mater. Interfaces 2020, 12, 33879-33886. [CrossRef]

31. Lepdatu, A.-M.; Slav, A.; Palade, C.; Dascalescu, I.; Enculescu, M.; Iftimie, S.; Lazanu, S.; Teodorescu, V.S.; Ciurea, M.L.; Stoica, T. Dense Ge nanocrystals embedded in $\mathrm{TiO}_{2}$ with exponentially increased photoconduction by field effect. Sci. Rep. 2018, 8, 4898. [CrossRef]

32. Ciurea, M.L.; Lepadatu, A.M. Tuning the properties of Ge and Si nanocrystals based structures by tailoring the preparation conditions. Dig. J. Nanomater. Biostructures 2015, 10, 59-87.

33. Stavarache, I.; Teodorescu, V.S.; Prepelita, P.; Logofatu, C.; Ciurea, M.L. Ge Nanoparticles in $\mathrm{SiO}_{2}$ for near infrared photodetectors with high performance. Sci. Rep. 2019, 9, 10286. [CrossRef] [PubMed]

34. Stavarache, I.; Lepadatu, A.M.; Maraloiu, A.V.; Teodorescu, V.S.; Ciurea, M.L. Structure and electrical transport in films of Ge nanoparticles embedded in $\mathrm{SiO}_{2}$ matrix. J. Nanoparticle Res. 2012, 14, 930. [CrossRef]

35. Alvarez-Diazcomas, A.; López, H.; Carrillo-Serrano, R.V.; Rodríguez-Reséndiz, J.; Vázquez, N.; Herrera-Ruiz, G. A Novel integrated topology to interface electric vehicles and renewable energies with the grid. Energies 2019, 12, 4091. [CrossRef]

36. Ray, S. A Quick review of machine learning algorithms. In Proceedings of the 2019 International Conference on Machine Learning, Big Data, Cloud and Parallel Computing (Com-IT-Con), Faridabad, India, 14-16 February 2019.

37. Pedregosa, F.; Varoquaux, G.; Gramfort, A.; Michel, V.; Thirion, B.; Grisel, O.; Blondel, M.; Prettenhofer, P.; Weiss, R.; Dubourg, V.; et al. Scikit-learn: Machine learning in Python. J. Mach. Learn. Res. 2011, 12, $2825-2830$.

38. Buitinck, L.; Louppe, G.; Blondel, M.; Pedregosa, F.; Mueller, A.; Grisel, O.; Niculae, V.; Prettenhofer, P.; Gramfort, A.; Grobler, J.; et al. API design for machine learning software: Experiences from the scikit-learn project. arXiv 2013; arXiv:1309.0238.

39. García-Martínez, J.R.; Cruz-Miguel, E.E.; Carrillo-Serrano, R.V.; Mendoza-Mondragón, F.; Toledano-Ayala, M.; Rodríguez-Reséndiz, J. A PID-type fuzzy logic controller-based approach for motion control applications. Sensors 2020, 20, 5323. [CrossRef] [PubMed]

Publisher's Note: MDPI stays neutral with regard to jurisdictional claims in published maps and institutional affiliations.

(C) 2020 by the authors. Licensee MDPI, Basel, Switzerland. This article is an open access article distributed under the terms and conditions of the Creative Commons Attribution (CC BY) license (http://creativecommons.org/licenses/by/4.0/). 